\title{
TIM PAWL
}

University of St. Thomas

\section{Paul Weingartner. God's Existence. Can it be Proven?: A Logical Commentary on the Five Ways of Thomas Aquinas. Frankfurt: Ontos Verlag, 2010.}

Paul Weingartner lists three tasks for this book:

1. "to show that the Five Ways of Thomas Aquinas can be presented in a form in which all five ways are logically valid arguments."

2. "to offer a detailed and critical discussion of the premises used in the arguments including important definitions used as premises."

3. "to examine the two preliminary questions of Thomas Aquinas, 'Whether the existence of God is self-evident?' and 'Whether it can be demonstrated that God exists"' (1).

The book has the following structure: the first two questions (from task 3 above) are presented then commented on extensively. The commentary on these two questions takes the reader halfway through this short book (116 pages, from cover to cover). In the final fifty pages of the book, each of the Five Ways is presented in the following manner. First the original Latin text is presented. Then the English translation from the Fathers of the English Dominican Province is given. At this point, for the sake of completing his first task, Weingartner takes the following two steps. "The first step is a preliminary interpretation under the title 'the premises and conclusions of the text' which attempts to concentrate all the relevant parts into natural language and making $[s i c]$ them as precise as possible. The second step translates this into the symbolic language of First Order Predicate Logic" (2). Finally, Weingartner provides a commentary on the terms, premises, and inferences of the Way in question. The book is well formatted, and it is easy to navigate.

We have seen some very helpful treatments of the Five Ways in the last decade or so. ${ }^{1}$ Unfortunately, this book does not rise to their level. In what follows, I will present three problems, along with illustrations of

${ }^{1}$ See, for instance, Bochenski, Joseph M. (2000) “The Five Ways" in The Rationality of Theism, ed. Adolfo García de la Sienra (Atlanta: Rodopi), 61-92; Wippel, John (2000), The Metaphysical Thought of Thomas Aquinas (Washington: Catholic University of America Press), Chapter 12. 
each. They are: (i) the commentary discusses too few topics, and does so in too shallow a manner; (ii) Weingartner's interpretation of many key concepts in the Five Ways is mistaken; (iii) the two-step translation process outlined above from the preliminary interpretation of the arguments to the First Order Predicate Logic (FOPL) reconstruction is done poorly.

Consider the first problem. Weingartner's second task in the book is "to offer a detailed and critical discussion of the premises" of the Ways. This is not done. Take the discussion of the First Way as an example. The commentary on the First Way is a mere five pages, with three of those five pages spent discussing how one should define the term "First Mover." There are only two other topics on which Weingartner comments. The first is whether step 6 in the formalization should be presented as following from prior premises, or presented as a premise itself. The second concerns the important question of how one should understand the meaning of the Latin word, movetur. The cluster of important questions concerning movetur receives little more than a page of commentary, though the questions here are myriad and complex. Should movetur be translated intransitively, as a one-place predicate- $\mathrm{x}$ is in motion-or passively, as a two-place predicate- $\mathrm{x}$ is moved by $\mathrm{y}$ ? What is the scope of motion in the First Way? Does he mean simply local motion? Or does it extend beyond local motion to qualitative and quantitative change as many claim? In addition to questions about the meaning of movetur, there are many other points where a reader desires commentary in the First Way. For instance, premise (4) says "Whatever moves the other is in actuality in that respect" (54). Beside the difficulties in phrasing here (the other what?; in what respect?), a common objection looms. There are many instances when something is not actual in a certain respect but moves things in that respect. What the reader needs here, and does not receive, is a commentary on how one ought to interpret this premise. Furthermore, since Weingartner reads movetur in the First Way as referring only to movement of physical bodies through space (see page 57), one wonders what sense it makes to talk about actuality in respect of movement across space. Even more difficult to understand is how God could be in actuality in respect of the movement of material bodies in space. And furthermore, even in mundane examples, I can push things rather than pulling them. That is, I can be potentially in my 
office, though push the chair to be actually in my office. I needn't pull the chair through the threshold of the door so that I am in actuality towards being in my office before my chair is. Commentary on this premise and the surrounding issues would have been helpful for the reader.

Similar points can be made for the other Ways. The Fifth Way, for instance, receives a mere two and a half pages of commentary (the Second Way receives the most commentary with eleven pages). On a related point, the commentary in the book is not well informed by the secondary literature on the Ways. The index lists fewer than five secondary sources that deal with the Five Ways in any detail, and only two works, from my count, that give a thorough examination of the Ways. The book would have greatly benefited from deeper and more prolonged commentary on the Ways.

The second difficulty I find in this book is that Weingartner's interpretation of some salient concepts of the Five Ways is faulty. For instance, I have already discussed his treatment of movetur in the First Way. Weingartner reads the scope of movetur narrowly, as only applying "to movement of material bodies in space" (57). The text, however, does not bear this interpretation out, since an example of motion that Aquinas uses in the very text of the First Way is that of fire moving wood to be hot, which is itself qualitative change, and not movement of a material body through space.

An example from the Second Way comes from Weingartner's interpretation of efficient causation. He says, writing about the premise "To take away the cause is to take away the effect", that "it should be underlined that it is very important that efficient cause is described or even defined there as a necessary condition" (66, Weingartner's italics). There are many problems with this interpretation of efficient causation. For instance, many premises in Weingartner's formulation come out false if Aquinas meant efficient causes to be defined as necessary conditions in this Way. Consider premise (2), which says "There is no case in which a thing is the efficient cause of itself" (62). This is false if being an efficient cause is defined as being a necessary condition in this argument, since any thing is, trivially, a necessary condition for itself. Likewise, premise (3), that "In efficient causes it is not possible to go on to infinity" is false, since mere necessary conditions can go on to infinity. Consider the series, "P is true;" "It is true that 'P is true;" "It is true 
that 'it is true that 'P is true;"' etc. Each is a necessary condition for the other(s), and the regress carries on infinitely. Furthermore, this example shows that efficient causation, if defined as merely being a necessary condition, can be circular, since two things can be necessary conditions for one another (as is the case with the right-hand and left-hand side of any true biconditional). For another example, which I do not have space to discuss here, see the understanding of 'maximum' in the Fourth Way.

The final difficulty with this book is that the two-step translation process outlined above from the reconstructions of the arguments to the First Order Predicate Logic (FOPL) is done poorly. They are not presented in a reader friendly way. It is sometimes unclear which premises from the first step are being reconstructed in the second step (see, for instance, premises $8 \mathrm{~b}$ and 14-16 of the Third Way, pg 77). The inference rule used to derive one line from others is sometimes cited but more often it is not, leaving the reader to try to figure out which rule, or rules, were employed to arrive at the line (see, for instance, the inference from 3 and 4 to 5 in the First Way, pg 55). This is a significant hindrance for the book, since the first task Weingartner sets himself in this book is to show that the proofs are deductively valid. To show this, it is not sufficient to name which premises are involved in the inference, the reader needs to know which deductively valid inference steps are employed as well. Weingartner uses numbers within parentheses (e.g., "(5b)") to number the lines of the first step, and uses numbers without parentheses (e.g., " $8 \mathrm{a}$ ") to number the lines of the FOPL translation, which can lead to some confusion for the reader. Finally, the definitions of the predicates employed in the reconstruction are listed at the end of the proof with little explanation.

The book contains numerous instances where the move from the first-step to the second-step fails. For example, see the step in the Third Way from the natural language premise (5b) to the FOPL translation 8a. Premise (5b):

(5b)because that which does not exist, only begins to exist by something already existing

Is translated as

$$
\text { 8a. } \exists t \forall x\left(\neg E X_{t} x\right) \rightarrow \neg \oslash \exists y \exists t(B E G y)
$$

where "EX $x \ldots x$ exists at $t\left(t_{0}=\right.$ present $)$ " and "BEG $y \ldots y$ begins at $t$ " (77). In English, 8a says that if there is a time at which nothing exists, 
then it is not possible for there to exist a thing and a time such that the thing begins to exist at that time. This is a poor translation of (5b). The same existential operator does not bind both times. And so the antecedent could be about one time and the consequent about another. For instance, suppose there exists a time, say, the first instant of the year $2700 \mathrm{AD}$ at which nothing exists. From this it would follow, given $8 \mathrm{a}$, that it is not possible for a thing to begin to exist at a time. But why would it be impossible for Napoleon to begin existing at a certain time on August $15^{\text {th }}, 1769$, given that almost a millennium later at the first instant of 2700AD nothing exists? The premise should tell the reader, instead, that if there is a time at which nothing exists, then at that very time, and for any time such that it is later than that time, it is not possible that something begin to exist. Moreover, note that even if 8 a were revised to avoid this translation error, it would still be a poor translation of (5b). (5b) tells us why it is that something cannot begin existing at times after which nothing exists (because something already existing is required to bring the new thing into existence). But 8a leaves out this vital reason.

Another example comes from the Fourth Way. Weingartner states the fourth premise of the Fourth Way (88):

(4)The maximum in any genus is the cause of all in that genus. His translation of this premise into FOPL reads (88):

7. $\forall z \forall r(M J z r f \rightarrow C z r u)$

where "MJxyf...x is greater than $y$ w.r.t. perfection" and "Czru...z causes $r$ w.r.t. being" (89). In English, 7 says that for any thing, $z$, and any thing, $r$, if $z$ is greater than $r$ with respect to perfection, then $z$ causes $r$ with respect to being. More colloquially, for any two things, if one is more perfect than the other, then the more perfect one causes the other to be. This is not a satisfactory translation of (4) into FOPL. As Weingartner notes (93),

the levels of perfection correspond to levels of being and the levels of being correspond to the actuality of essential forms. Therefore a rough division of levels of perfection is this: (a) non-living beings, (b) living beings without senses, (c) animals, (d) human beings, (e) angels, (f) God.

So some human, Bob, is more perfect than any non-living thing, any plant, and any non-rational animal. By $\forall$ elimination on 7 (substituting Bob for $z$ ), we can derive, in English, that for any $r$, if Bob is more perfect 
than $r$, Bob is the cause of $r$. Then, noting Bob's level of perfection, we can substitute in any animal or plant for $r$ and yield that Bob causes that animal or plant. So, by 7 , each individual human is the cause of each individual non-living thing, plant, and non-rational animal. (4) does not have this unfortunate entailment, and so 7 is not a satisfactory translation of 4 into FOPL. The error is with using two universal quantifiers in this way. This same type of error appears in premises 5 and 6 of the argument as well (88). A similar error appears in the translation from (1) to 1 of the Fifth Way (95). This error, like the error in Way Four, infects other premises in the argument (premises 2-4).

In summary, while the book is carefully laid out and there are some parts of interest, Weingartner's commentaries don't cover enough material, his understanding of some major concepts in the Five Ways is flawed, and his formalizations contain serious errors. ${ }^{2}$

${ }^{2}$ I thank David Clemenson, Marie Feldmeier, Sandra Menssen, Yujin Nagasawa, Michael Rota and Jonathan Stoltz for their aid in composing this review. 